\title{
Early time optical spectroscopy of supernova SN 1998S
}

\author{
G. C. Anupama, T. Sivarani, and G. Pandey \\ Indian Institute of Astrophysics, II Block Koramangala, Bangalore 560 034, India \\ Received 26 June 2000 / Accepted 4 December 2000

\begin{abstract}
We present medium-resolution optical spectra of the type II supernova SN 1998S obtained during the pre-maximum, maximum and early decline phases. The early decline spectra show complex Balmer line profiles: a narrow P-Cygni emission feature superimposed on a broad emission. The narrow emission has a redshift similar to that of the host galaxy, indicating its origin from a pre-supernova circumstellar material. The H $\alpha$ luminosity of the broad component implies a shell mass of $\sim 0.1 M_{\odot}$ for the supernova ejecta, while the luminosity of the narrow component implies a mass loss rate of $10^{-4} M_{\odot} \mathrm{yr}^{-1}$ for the precursor. The photospheric temperature of the supernova shell decreased from a value of $18400 \pm 2900 \mathrm{~K}$ on day 17 to a value of $7500 \pm 1200 \mathrm{~K}$ on day 44. The observations indicate SN 1998S belongs to type IIn supernovae where the supernova ejecta dynamically interact with a pre-supernova circumstellar material.
\end{abstract}

Key words. supernovae: general - supernovae: individual (SN 1998S)

\section{Introduction}

The supernova SN 1998S was discovered in the SCII.2 galaxy NGC 3877 on 1998 March 2.68 by Z. Wan at the Beijing Astronomical Observatory, at a discovery magnitude of 15.2 ( $\mathrm{Li} \& \mathrm{Li}$ 1998; Qiu 1998a). The object had brightened to about $13.5 \mathrm{mag}$ on March 4.3 indicating a pre-maximum discovery. Figure 1 shows the visual light curve of SN 1998S based on daily visual magnitude estimates from VSNET. CCD photometry is presented by Fassia et al. (2000) and Liu et al. (2000). Based on the visual light curve, we estimate the optical maximum reached around 1998 March 20 with a visual maximum of 11.8 mag. A pre-discovery frame obtained on 1988 February 23.7 shows no evidence of the supernova, down to a limiting magnitude of $\sim+18$ (Qiu 1998a; Leonard et al. 2000). Following Leonard et al. (2000) and Fassia et al. (2000), we adopt the discovery date of March 2.68 as Day 0 , the day of shock breakout. The optical maximum thus occurred at $\sim$ Day 18 . Early spectra of the supernova (Filippenko \& Moran 1998a, 1998b) indicated the supernova was of type IIn.

The subclass IIn supernovae (Schlegel 1990; Filippenko 1997) are those supernovae in which the broad absorption components of all lines are weak or absent. Their spectra are dominated by strong emission lines that have a complex profile. The hydrogen emission lines typically exhibit a narrow component $\left(F W H M \sim 200-300 \mathrm{~km} \mathrm{~s}^{-1}\right)$ superposed on an intermediate $\left(F W H M \sim 1000-2000 \mathrm{~km} \mathrm{~s}^{-1}\right)$

Send offprint requests to: G. C. Anupama,

e-mail: gca@iiap.ernet.in or broad ( $\left.F W H M \sim 5000-10000 \mathrm{~km} \mathrm{~s}^{-1}\right)$ base. The centroid of the base is blue-shifted relative to the narrow portion of the profile. The continuum is bluer than normal during the early stages. The early time spectra are dominated by strong narrow emission lines. The broad emission base arises from the supernova ejecta, while the intermediate and narrow lines arise in the circumstellar material emitted by the progenitor (e.g. Chugai 1991; Turatto et al. 1993; Chugai \& Danziger 1994). The intermediate lines arise in the region of the dynamical interaction of the SN envelope with the circumstellar material, while the narrow emission lines arise in the undisturbed circumstellar material photoionized by the SN event. The evolution of the spectrum is slow. All the known type IIn supernovae have appeared in spiral galaxies.

Leonard et al. (2000) present spectroscopic observations of SN 1998S spanning the first 494 days after discovery, and spectropolarimetric observations made 5 days after discovery. A high degree of linear polarization was detected implying significant asphericity for the continuumscattering medium. The later time spectra exhibit broad, structured emission-line profiles, implying a disc-like or ring-like morphology for the dense circumstellar medium of the progenitor. Leonard et al. also suggest that the progenitor of SN 1998S underwent two mass-loss episodes. One that ended 60 years prior to the explosion, during which mass loss was significant, and a second, weaker mass-loss episode that began about 7 years prior to the explosion.

Fassia et al. (2000) present the optical and infrared photometry covering $\sim 150$ days after the discovery. 


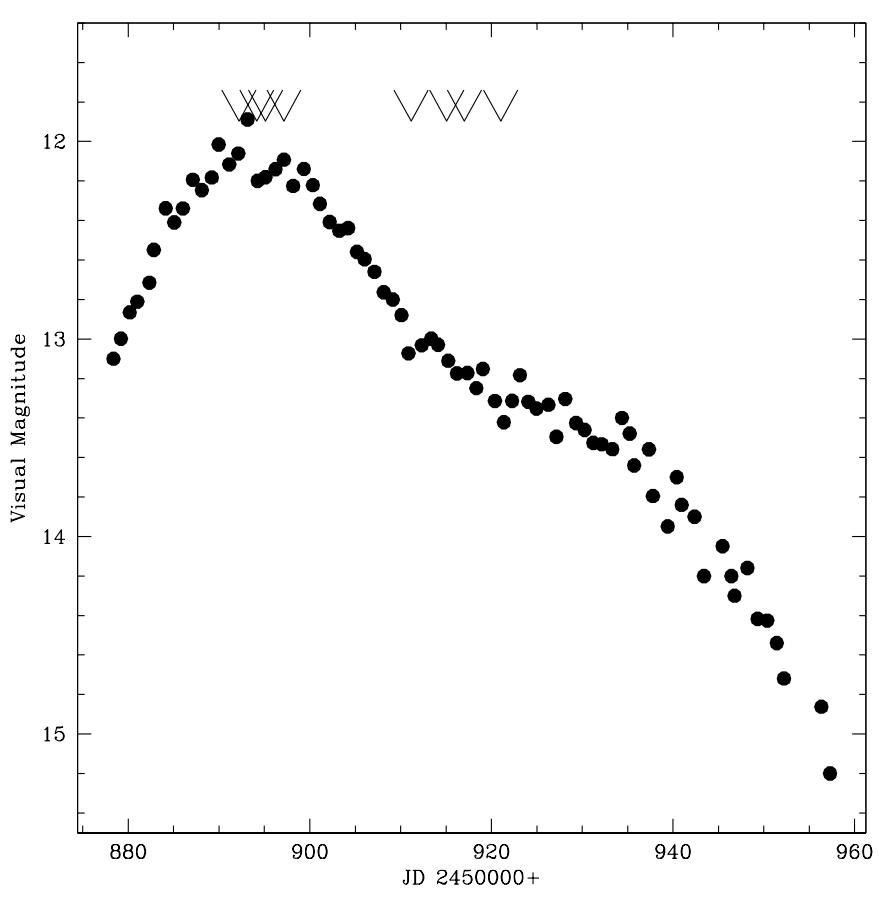

Fig. 1. Visual light curve of SN 1998S. The dates of VBO observations are marked

They report the detection of a strong IR excess $\left(K-L^{\prime}=\right.$ $2.5)$ on day 130 and interpret this as due to thermal emission from dust grains located in the circumstellar medium. Liu et al. (2000) present optical photometric and spectroscopic evolution through the first four months.

We present here optical spectra of the supernova obtained from the Vainu Bappu Observatory (VBO), Kavalur, India, during 1998 March 13-April 15.

\section{Observations and reductions}

Optical CCD spectra were obtained using the OMR spectrograph on the 2-m VBT (Prabhu et al. 1998) at VBO on March 13.82, March 17.80, March 19.79, March 20.75, March 22.77, April 5.78, April 9.70, April 11.66 and April 15.65. All dates are in UT. All spectra were obtained with a slit of 2 arcsec width aligned at $0^{\circ} \mathrm{PA}$. The spectroscopic standard Feige 34 was observed on each night with a slit of 5 arcsec width. The observations covered the premaximum, maximum and the early decline phases. Table 1 gives the journal of observations. The dates of VBO observations are marked in Fig. 1.

All spectra were bias subtracted, flat-field corrected, extracted and wavelength calibrated in the standard manner using the IRAF ${ }^{1}$ reduction package. The spectra were corrected for instrumental response and brought to a relative flux scale using the spectrophotometric standard Feige 34 observed on the same night. Since the supernova spectra were obtained with a narrow slit, and the sky conditions were generally non-photometric, the fluxes obtained are not on an absolute flux scale. However, a

${ }^{1}$ IRAF is distributed by National Optical Astronomy Observatories, USA.
Table 1. Journal of observations from VBO

\begin{tabular}{rrlcrr}
\hline $\begin{array}{r}\text { Date } \\
\text { (UT) }\end{array}$ & $\begin{array}{c}\text { Epoch* } \\
(\text { Days })\end{array}$ & Phase & $\begin{array}{c}\text { Wavelength } \\
\AA\end{array}$ & $\begin{array}{c}\text { Resln } \\
\AA\end{array}$ & Airmass \\
\hline March & & & & & \\
13.82 & 11.14 & pre-max & $4200-5800$ & 2.6 & 1.01 \\
17.80 & 15.12 & pre-max & $4500-5700$ & 11.0 & 1.08 \\
19.79 & 17.11 & $\max$ & $3500-7000$ & 11.0 & 1.22 \\
20.75 & 18.07 & $\max$ & $3500-9500$ & 11.0 & 1.27 \\
22.77 & 20.09 & $\max$ & $3500-9500$ & 11.0 & 1.22 \\
& & & & & \\
April & & & & & \\
5.78 & 34.10 & decline & $4600-7200$ & 5.5 & 1.26 \\
9.70 & 38.02 & decline & $3900-5000$ & 2.6 & 1.22 \\
& & & $5500-6800$ & 2.6 & 1.24 \\
11.66 & 39.98 & decline & $5800-7000$ & 2.6 & 1.29 \\
15.65 & 43.97 & decline & $4000-9200$ & 11.0 & 1.29 \\
\hline
\end{tabular}

* Days since 1998 March 2.68.

zero-point correction has been applied to the fluxes based on the $V$ band magnitudes from VSNET.

\section{The spectral evolution}

\subsection{Pre-maximum phase}

The early spectra of SN 1998S (Filippenko \& Moran 1998a, 1998b) showed prominent hydrogen emission lines superposed on a featureless blue continuum. Helium emission lines were also seen in the spectra. The emission lines showed a narrow peak with broad wings. Strong He II $4686 \AA$ and permitted N III/C III $4640 \AA$ were also seen (Garnavich et al. 1998). Several other emission features characteristic of Wolf-Rayet stars, such as the C IV $5800 \AA$ and the C II blend at $7100 \AA$ were also present in the spectrum on March 5.3 (Garnavich et al. 1998) and March 7.7 (Qiu 1998b). The spectrum in general resembled that of the pre-maximum spectrum of SN 1983K (Niemela et al. 1985). No P-Cygni absorption was detected at this phase. By March 12, the Wolf-Rayet features had almost disappeared, the Balmer profiles changed to be asymmetric with steeper blue wings and had also weakened in strength (Qiu 1998b).

The VBO spectrum of March 13.82 (day 11.14) shows a blue continuum with a weak $\mathrm{H} \beta$ emission (Fig. 2). The $\mathrm{H} \beta$ line profile is similar to the profile of March 12 .

\subsection{Maximum phase}

The spectrum during this phase shows a blue continuum superposed with Balmer emission lines. The emission line profile had changed by March 17.8 (day 15.12). The $\mathrm{H} \beta$ line showed a narrow P-Cygni profile and a blue shifted broad, shallow absorption trough. The profile is similar to 


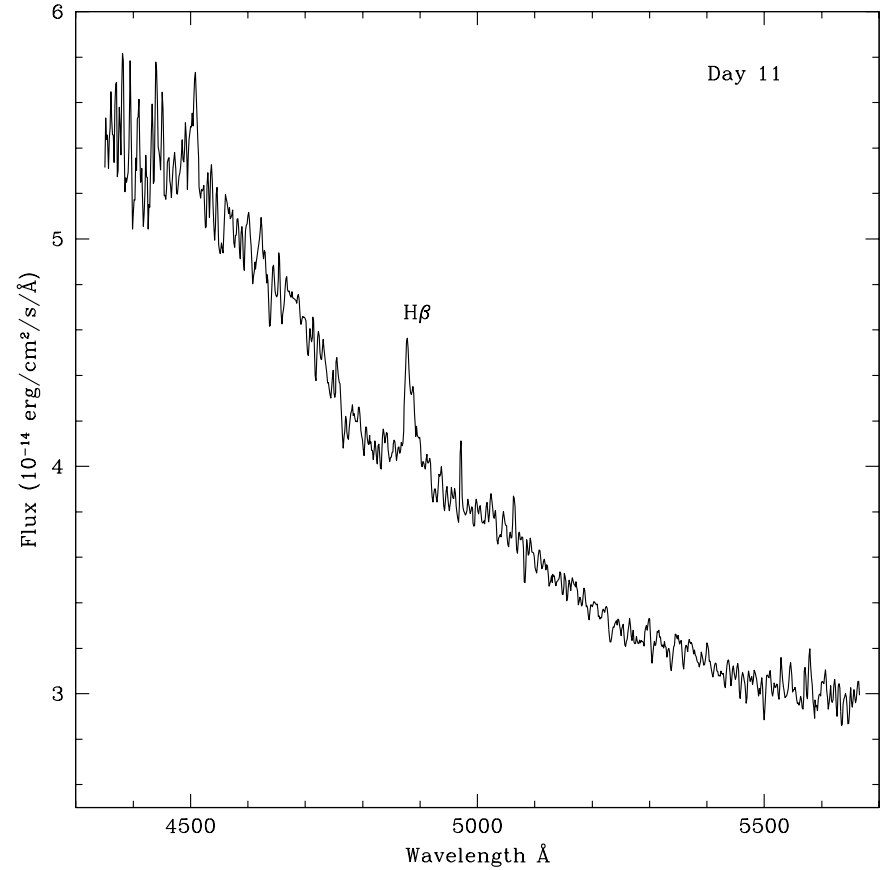

Fig. 2. Spectrum of SN 1998S obtained on Day 11. Dates are relative to date of discovery, 1998 March 2.68. Note that a) the spectra have not been corrected for the galaxy redshift; b) fluxes are not on an absolute scale, in this figure and also in Figs. 3-5

that of March 16 (Garnavich et al. 1998). A similar profile was seen in the $\mathrm{H} \alpha$ line also.

Figure 3 shows the spectrum on March 19.79, 20.75 and 22.77; days $17.11,18.37$ and 20.09 respectively. Fe II 5018, $5169 \AA$ absorption features began to develop by March 19.

\subsection{Early decline}

The Fe II absorption features increased in strength with time. The $\mathrm{H} \beta$ line was blended with Fe II lines by April 5.78 (day 34.1). By this time, the narrow P-Cyg feature had disappeared in the $\mathrm{H} \beta$ line, while still present in $\mathrm{H} \alpha$ and continued to be present until April 15.65 (day 43.97). The P-Cyg absorption was not present in $\mathrm{H} \alpha$ also on this day, although the narrow emission component continued to be present.

Spectra during this period are plotted in Fig. 4.

Absorption features due to He I $5876 \AA$, Fe II 5018, $5169 \AA$, Si II $6350 \AA$ and Sc II 5527, $5658 \AA$ identified by Leonard et al. (2000) are clearly present in the spectra during this phase.

The spectrum of April 15.65 (43.97) also shows the O I $8446 \AA$ and CaII IR triplet lines in the $8500-8600 \AA$ region.

\section{4. $H \alpha$ profile}

The $\mathrm{H} \alpha$ line developed a broad profile $(F W H M \sim$ $4000 \mathrm{~km} \mathrm{~s}^{-1}$ ) around April 5 (34.10). The centroid of the

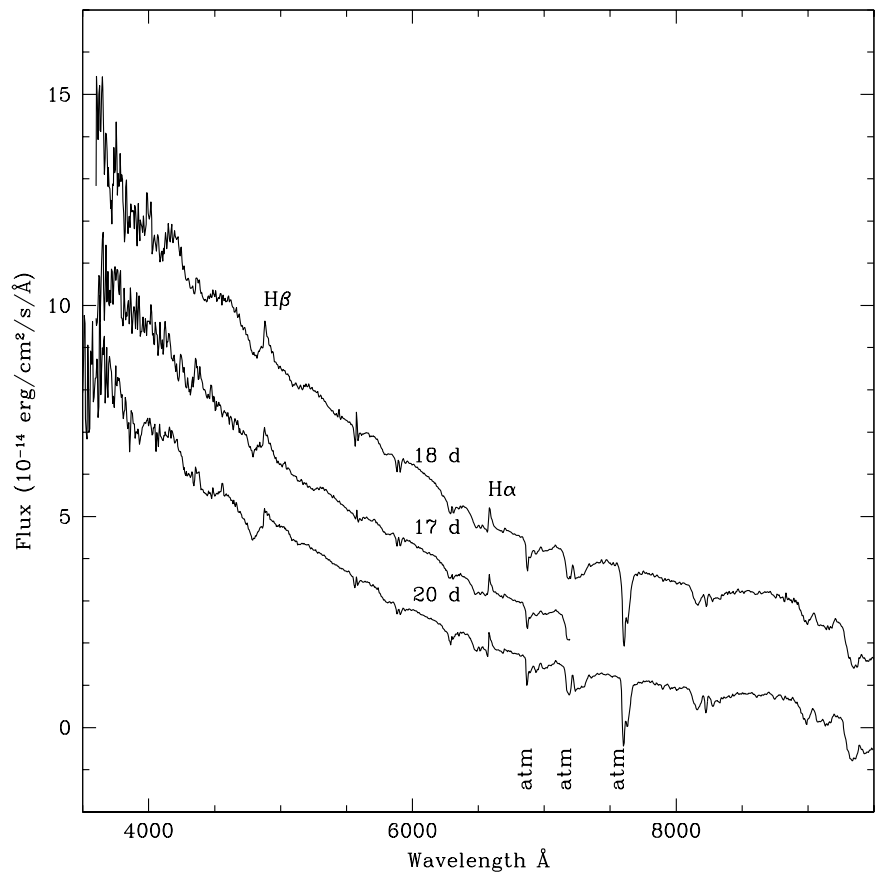

Fig. 3. Spectrum of SN 1998S obtained on days 17, 18 and 20. Constants of 1 and -1 have been added to fluxes of day 18 and 20 , respectively

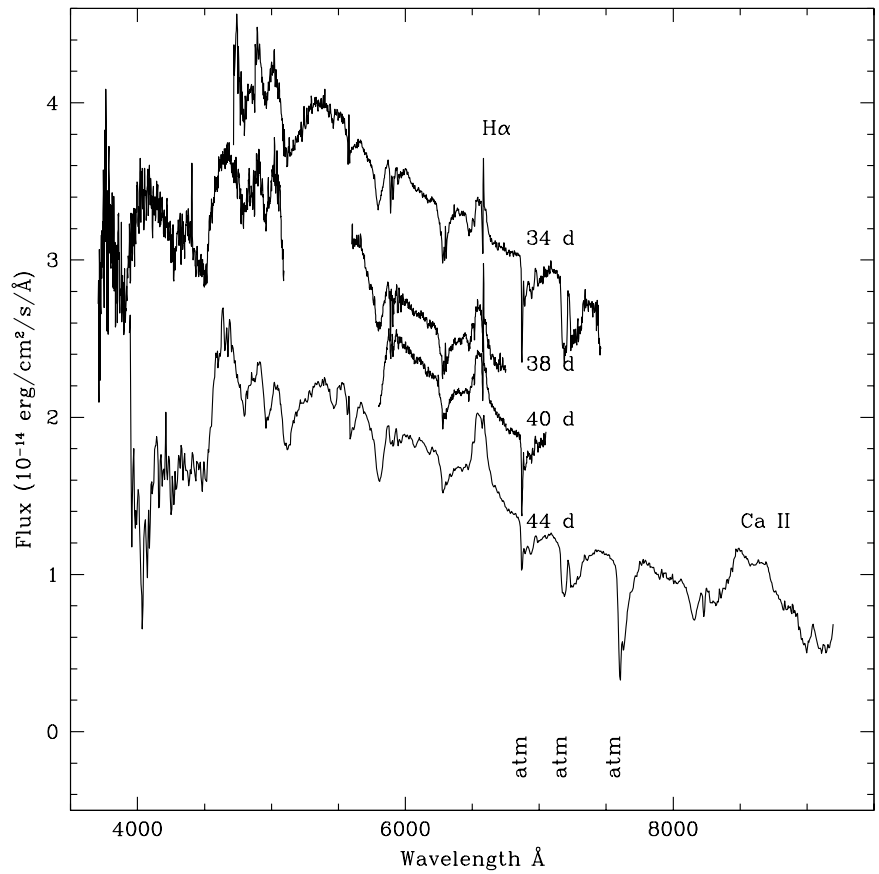

Fig. 4. Spectrum of SN 1998S obtained on days 34, 38, 40 and 44. Constants 1, 0.8 and 0.5 have been added to fluxes of day 34,38 and 40 , respectively

broad emission was blue shifted by $\approx 900 \mathrm{~km} \mathrm{~s}^{-1}$ with respect to the galaxy, similar to that seen in other supernovae of type IIn (Schlegel 1990). The narrow P-Cygni feature was superposed on this broad feature. The narrow emission peak has a velocity $+890 \mathrm{~km} \mathrm{~s}^{-1}$ similar to galaxy redshift, implying it originates from the pre-supernova circumstellar shell. Branch et al. (2000) have shown that 


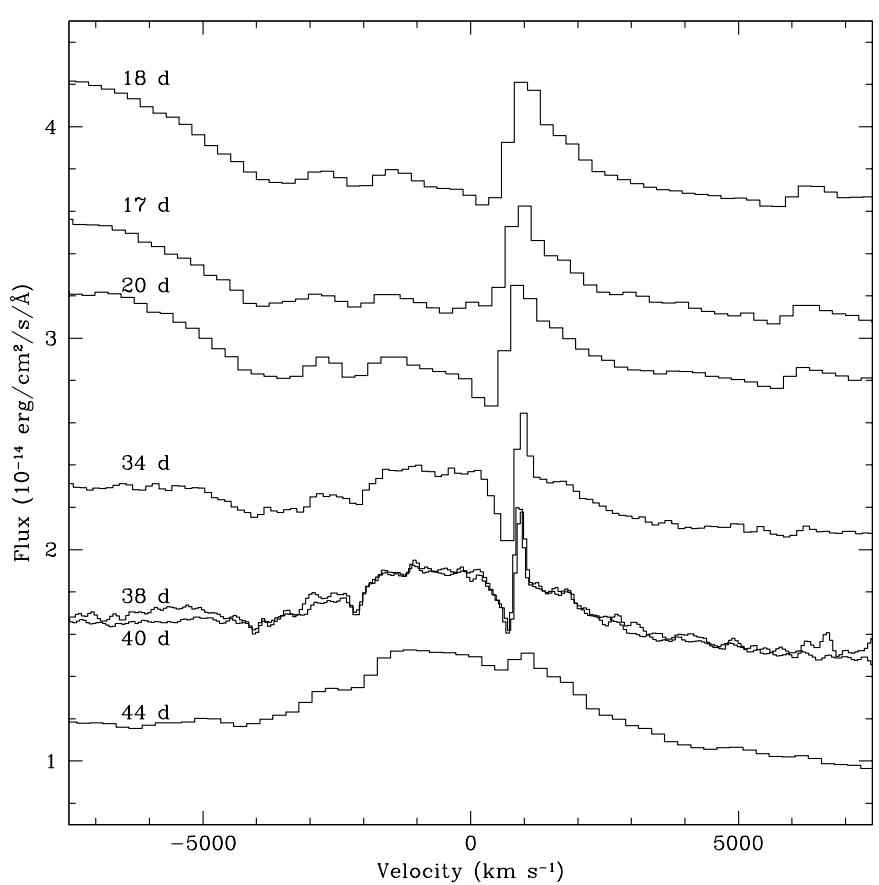

Fig. 5. Temporal evolution of the $\mathrm{H} \alpha$ line profile based on VBO data. Constant -0.5 has been added to the fluxes of day 44

"toplighting", or illumination of the broad-line forming region from the region of circumstellar interaction, in addition to the photosphere, can lead to a blue shifted emission peak with absorption at the line centre wavelength (a flipped P-Cygni profile). They suggest the presence of this effect in SN 1998S and also that some of the P-Cygni profiles in the early times may be flipped.

Figure 5 shows the temporal development of the $\mathrm{H} \alpha$ line profile.

\section{Discussion}

\subsection{Absorption velocities}

The maximum absorption velocities were measured from the blue edge of the broad absorption troughs of $\mathrm{H} \beta, \mathrm{H} \alpha$ and Si II $6350 \AA$. The mean velocity decreased from a value of $-10040 \mathrm{~km} \mathrm{~s}^{-1}$ on day 11.14 to $-5540 \mathrm{~km} \mathrm{~s}^{-1}$ on day 43.97. Figure 6 shows the evolution of the $\mathrm{H} \beta$ and $\mathrm{H} \alpha$ velocities.

The velocity of the narrow component measured based on the minimum of the P-Cyg absorption in $\mathrm{H} \alpha$ decreased from $-700 \mathrm{~km} \mathrm{~s}^{-1}$ on day 17 to $-230 \mathrm{~km} \mathrm{~s}^{-1}$ on day 40 . The maximum velocity corresponding to this component was $\sim-2400 \mathrm{~km} \mathrm{~s}^{-1}$ on days 17-20. The corresponding $\mathrm{H} \beta$ velocity was $-500 \mathrm{~km} \mathrm{~s}^{-1}$ on day 15 .

The absorption velocities are listed in Table 2 .

\subsection{Spectral continuum and photospheric temperature}

The continuum colours are estimated based on the spectral continuum magnitudes obtained using

$m_{\lambda}=-2.5 \log F_{\lambda}-21.1$,

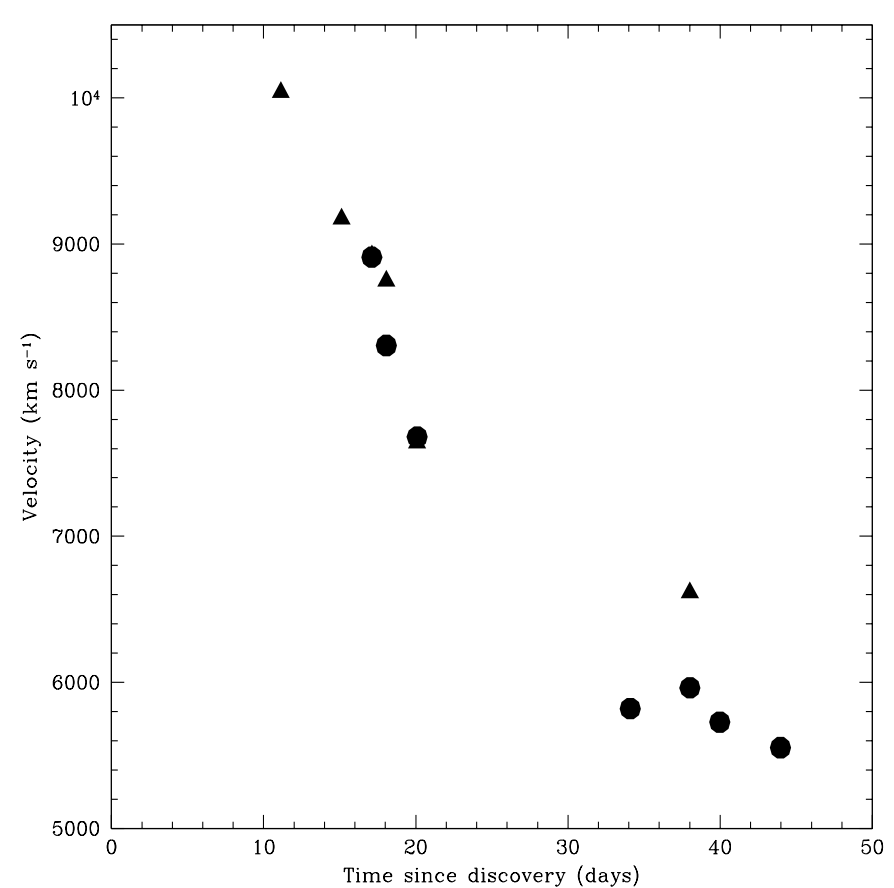

Fig. 6. Development of the $\mathrm{H} \beta$ (filled triangles) and $\mathrm{H} \alpha$ (filled circles) broad absorption trough velocities based on VBO data

Table 2. Blue shifted absorption velocities

\begin{tabular}{llccl}
\hline $\begin{array}{l}\text { Epoch } \\
\text { Days }\end{array}$ & \multicolumn{5}{c}{ Absn velocities $\left(\mathrm{km} \mathrm{s}^{-1}\right)$} \\
& $\mathrm{H} \beta$ & $\mathrm{H} \alpha$ & $\mathrm{Si} \mathrm{II}$ & $\mathrm{H} \alpha$ \\
\hline 11.14 & 10038 & & & \\
15.12 & 9172 & & & $500^{*}$ \\
17.11 & 8921 & 8910 & 9070 & 700 \\
18.07 & 8749 & 8306 & & 680 \\
20.09 & 7639 & 7680 & 7654 & 600 \\
34.10 & & 6030 & 6570 & 270 \\
38.02 & & 5990 & 6240 & 230 \\
39.98 & & 5728 & 5763 & 230 \\
43.97 & & 5553 & 5530 & \\
\hline
\end{tabular}

1: Based on absorption minimum.

$* \mathrm{H} \beta$.

where $F_{\lambda}$ is the observed flux averaged over a $20 \AA$ bin centered at $4000 \AA(b), 5000 \AA(v), 6000 \AA(r)$ and $7000 \AA$ $(i)$. The estimated continuum magnitudes and colours are tabulated in Table 3 . The continuum which was extremely blue during the pre-maximum and maximum phases got redder with time.

The continuum fluxes may be used to make an estimate of the photospheric temperature and radius. However, the shape of the continuum could be affected due to improper flux calibration (see Sect. 2). We correct for this using zero points based on $U B V R I$ photometry available in the literature (Fassia et al. 2000; Liu et al. 2000). Zero points for the entire observed spectra could be obtained only for days 
Table 3. Spectral continuum magnitudes, colours and photospheric temperature

\begin{tabular}{llllllllrr}
\hline Epoch & \multicolumn{4}{c}{ Magnitude $^{1}$} & \multicolumn{3}{c}{ Colour $^{1}$} & $T_{\mathrm{pp}}$ \\
Days & $b$ & $v$ & $r$ & $i$ & $b-v$ & $v-r$ & $r-i$ & $\mathrm{~K}$ \\
\hline 11.14 & & 12.45 & & & & & & \\
15.12 & & 11.92 & & & & & & \\
17.11 & 11.44 & 11.91 & 12.30 & 12.82 & -0.47 & -0.39 & -0.53 & $18400 \pm 2900$ \\
18.07 & 11.28 & 11.71 & 12.10 & 12.64 & -0.43 & -0.39 & -0.54 & \\
20.09 & 11.61 & 12.00 & 12.45 & 12.95 & -0.39 & -0.45 & -0.50 & $14000 \pm 2250$ \\
34.10 & & 12.63 & 12.87 & 13.23 & & -0.24 & -0.36 & $10400 \pm 1550$ \\
$39.0^{*}$ & 12.88 & 12.83 & 13.18 & 13.60 & 0.05 & -0.35 & -0.42 & \\
43.97 & 13.50 & 13.11 & 13.21 & 13.69 & 0.30 & -0.10 & -0.48 & $7500 \pm 1200$ \\
\hline
\end{tabular}

1: Observed magnitudes and colours not corrected for reddening.

See text for definition of the magnitudes.

*: Average of days 37.02 and 38.98 .

$17.11,20.09,34.10$ and 43.97. Hence, only these spectra are calibrated for absolute fluxes and used to make an estimate of the photospheric temperature. The spectra were corrected for reddening using a value of $E(B-V)=0.22$ (Fassia et al. 2000). A low order cubic spline, ignoring the absorption and emission lines, was fit to the continuum. A blackbody function was fit to the continuum using the IRAF nfit1d task. In Fig. 7, we plot the observed, reddening corrected spectra and the best fit blackbody function for days 17.11, 20.09, 34.10 and 43.97. The best fit blackbody curves indicate the photospheric temperature fell from $18400 \pm 2900 \mathrm{~K}$ on day 17.11 to $7500 \pm 1200 \mathrm{~K}$ on day 43.97. The estimated photospheric temperatures are listed in Table 3. The estimates for days 17.11, 20.09 and 34.10 are higher than the photospheric temperatures obtained by Fassia et al. (2000) using $U B V R I J H K$ fluxes, while the estimate for day 43.97 is similar to the estimate by Fassia et al.

\subsection{Physical parameters of the shell(s)}

The mass and density of the expanding SN shell can be computed assuming homologous expansion and knowing its density in one layer. One method of estimating the density is using the luminosity of the $\mathrm{H} \alpha$ component (Osterbrock 1989)

$L_{\mathrm{H} \alpha}=\left(\frac{4 \pi j_{\mathrm{H} \alpha}}{N_{\mathrm{e}}^{2}}\right) N_{\mathrm{e}}^{2} V$,

where the term in the bracket represents the $\mathrm{H} \alpha$ emissivity.

Assuming the broad component is due to the expanding SN shell, we estimate the shell mass. The observed, reddening corrected $\mathrm{H} \alpha$ (broad) fluxes were measured for days 37.37 (Average of days 34.10, 38.02 and 39.98 ) and 43.97 as $3.710^{-13} \mathrm{erg} \mathrm{cm}^{-2} \mathrm{~s}^{-1}$ and $8.710^{-13} \mathrm{erg} \mathrm{cm}^{-2} \mathrm{~s}^{-1}$, respectively. These fluxes were converted to luminosity $L_{\mathrm{H} \alpha}^{\mathrm{b}}$ adopting a distance of
17.0 Mpc to NGC 3877 (Tully 1988). The volume $V$ was estimated assuming an expansion velocity of $10000 \mathrm{~km} \mathrm{~s}^{-1}$. The $\mathrm{H} \alpha$ emissivity was obtained from Hummer \& Storey (1987) for a temperature of $10^{4} \mathrm{~K}$. Using these values, we obtain $N_{\mathrm{e}} \approx 810^{8} \mathrm{~cm}^{-3}$. Assuming the photosphere lies at the base of the broad-line-forming region (Wegner \& Swanson 1996), we estimate the shell mass as $M_{\text {shell }} \sim 0.1 M_{\odot}$. This estimate for the shell mass, which is a lower limit, is similar to that estimated for the type IIn SN 1987F (Wegner \& Swanson 1996).

The early as well as the late spectra of SN 1998S indicate a shock interaction of the SN shell with a dense circumstellar medium (CSM) (Leonard et al. 2000). Based on shock interaction models (e.g. Chevaliar 1990), the density in the CSM outside the shock region is given by

$\rho_{c}=\frac{1}{4 \pi r^{2}}\left(\frac{\dot{M}}{v_{\mathrm{w}}}\right)$,

where $\dot{M}$ is the precursor mass loss rate and $v_{\mathrm{w}}$ is the wind velocity. The radius $r$ of the CSM may be obtained using a wind velocity of $10 \mathrm{~km} \mathrm{~s}^{-1}$ and assuming the mass loss occurred 60 years prior to the outburst (Leonard et al. 2000). Using the $\mathrm{H} \alpha$ luminosity in the narrow component, $L_{\mathrm{H} \alpha}^{\mathrm{n}}=1.210^{39} \mathrm{erg} \mathrm{s}^{-1}$ as observed on day 37.37 to estimate the density, we obtain $\dot{M} / u_{\mathrm{w}} \sim 7.510^{15} \mathrm{~g} \mathrm{~cm}^{-1} \mathrm{~s}^{-1}$, or $\dot{M} \sim 10^{-4} M_{\odot} \mathrm{yr}^{-1}$ for $v_{\mathrm{w}}=10 \mathrm{~km} \mathrm{~s}^{-1}$. This estimate for $\dot{M}$, although lower than that of SN 1987F (Chugai 1991 ) is similar to the estimate for SN 1984E (Dopita et al. 1984).

\subsection{Comparison with other supernovae}

The early spectra of SN $1988 \mathrm{~S}$ resembled that of SN 1983K. The pre-maximum spectra showed broad Balmer and high excitation Wolf-Rayet features (Leonard et al. 2000; Liu et al. 2000). The Wolf-Rayet features disappeared around maximum, by which time the spectrum 

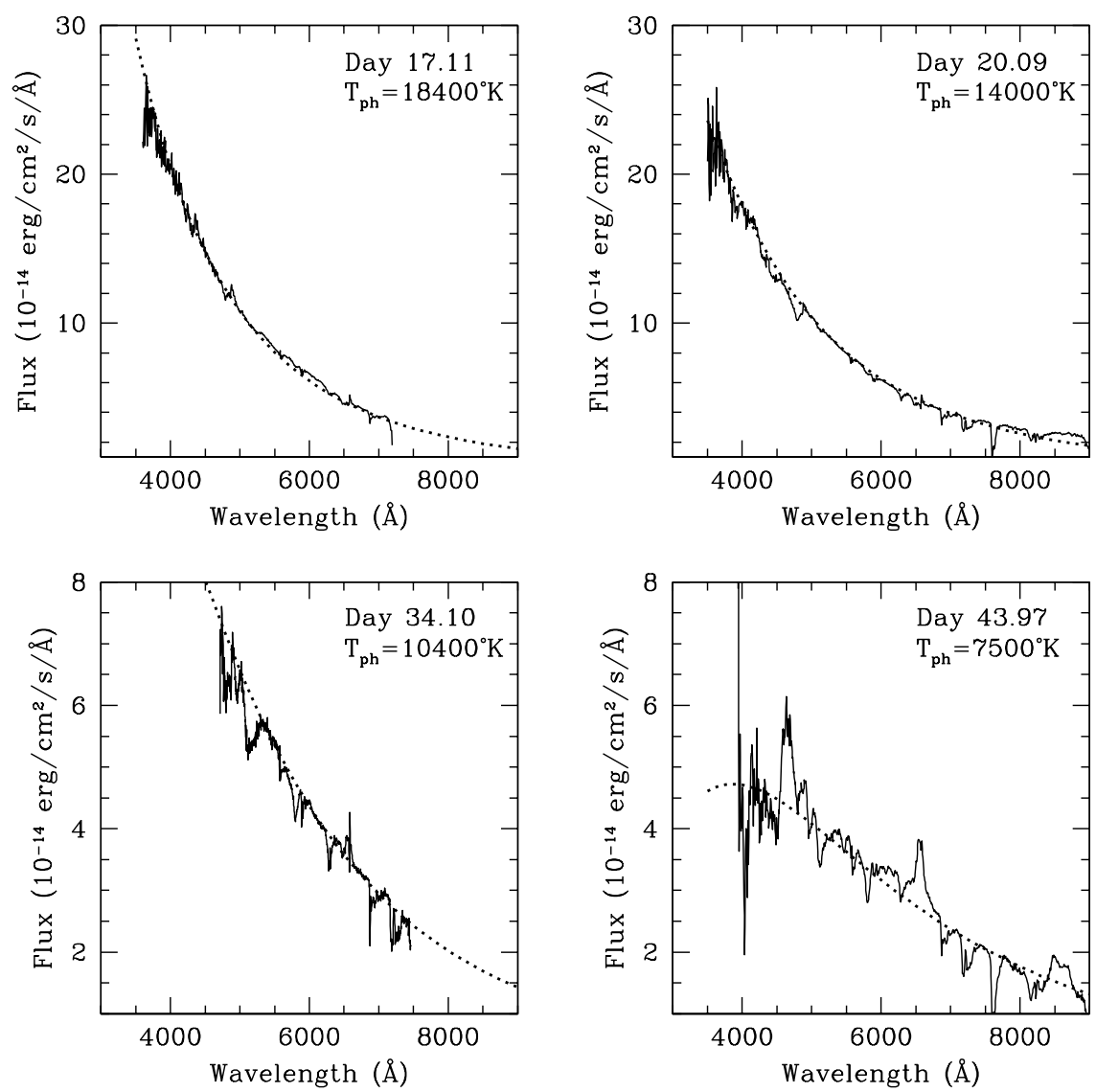

Fig. 7. Reddening corrected observed spectra (solid line). The best fit blackbody curve is also plotted (dotted line), and the corresponding photospheric temperature marked

had changed to a featureless blue continuum with weak Balmer lines as in the case of SN 1983K. The spectra of SN 1998S around maximum resemble that of SN 1983K about 24 days past maximum.

The mass of the ejected $\mathrm{SN}$ shell is $\sim 0.1 M_{\odot}$. This value is similar to that estimated for SN $1987 \mathrm{~F}\left(\sim 0.2 M_{\odot}\right.$ : Wegner \& Swanson 1996) and SN $1988 Z\left(0.1-0.5 M_{\odot}\right.$ : Chugai \& Danziger 1994).

The spectrum of SN $1984 \mathrm{E} \approx 2$ days after discovery (Dopita et al. 1984) is very similar to those of SN 1998S during days 33-39 (Fig. 4). The $\mathrm{H} \beta$ and $\mathrm{H} \alpha$ line profiles in both supernovae are also similar with a narrow P-Cyg profile superimposed over a broad profile. The precursor mass loss rate of $\sim 10^{-4} M_{\odot} \mathrm{yr}^{-1}$ estimated for SN 1998S is similar to SN 1984E, but lower than SN 1988Z (Chugai 1991). This mass loss rate corresponds to the first massloss episode suggested by Leonard et al. (2000).

It thus appears that SN $1998 \mathrm{~S}$ compares well, albeit with differences, with other type IIn supernovae, or those supernovae which have shown evidences for the interaction of the SN ejecta with a pre-existing CSM.

\section{Summary}

The temporal development of the spectrum of SN 1998S is presented here. The presence of narrow hydrogen lines indicate the supernova was of type IIn. The continuum was extremely blue and featureless close to the optical maximum and got redder with time. Fe II absorption features began to develop and strengthen with time.

During the early decline phase, the Balmer lines showed a narrow P-Cyg emission superimposed over a broad profile. Based on the $\mathrm{H} \alpha$ luminosity in the broad component, the ejected SN shell mass is estimated as $\sim 0.1 M_{\odot}$. The $\mathrm{H} \alpha$ luminosity of the narrow component indicates a mass loss rate of $\sim 10^{-4} M_{\odot} \mathrm{yr}^{-1}$ for a wind velocity of $10 \mathrm{~km} \mathrm{~s}^{-1}$.

SN 1998S thus clearly belongs to the class of type IIn supernovae where the supernova ejecta dynamically interacts with a pre-supernova circumstellar material formed by mass-loss episode(s) in the precursor.

Acknowledgements. We thank Mr. G. Selvakumar for obtaining part of the data. We thank the referee Dr. D. C. Leonard for several useful suggestions and comments.

\section{References}

Branch, D., Jeffery, D. J., Blatlock, M., \& Hatano, K. 2000, PASP, 112, 217

Chevalier, R. A. 1990, in Supernovae, ed. A. J. Petschek, A\&A, Library (Springer-Verlag: New York), 91

Chugai, N. N. 1991, MNRAS, 250, 513 
Chugai, N. N., \& Danziger, I. J. 1994, MNRAS, 173, 180

Dopita, M. A., Evans, R., Cohen, M., et al. 1984, ApJL, 287, L69

Fassia, A., Meikle, W. P. S., Vacca, W. D., et al. 2000, MNRAS, 318,1093

Filippenko, A. V. 1997, ARA\&A, 35, 309

Filippenko, A. V., \& Moran, E. C. 1998a, IAUC, 6829

Filippenko, A. V., \& Moran, E. C. 1998b, IAUC, 6830

Garnavich, P., Jha, S., \& Kirshner, R. 1998, IAUC, 6832

Garnavich, P., Kirshner, R., \& Challis, P. 1998, IAUC, 6845

Hummer, D. G., \& Storey, P. J. 1987, MNRAS, 224, 801

Leonard, D. C., Filippenko, A. V., Barth, A. J., et al. 2000, ApJ, 536, 239

Li, W.-d., \& Li, C. 1998, IAUC, 6829

Liu, Q.-Z., Hu, J.-Y., Hang, H.-R., et al. 2000, A\&AS, 144, 219
Niemela, V. S., Ruiz, M. T., \& Phillips, M. M. 1985, ApJ, 289, 52

Osterbrock, D. E. 1989, Astrophysics of Gaseous Nebulae and Active Galactic Nuclei (Mill Valley: University Science Books)

Prabhu, T. P., Anupama, G. C., \& Surendiranath, R. 1998, Bull. Astr. Soc. India, 26, 383

Qiu, Y. L. 1998a, IAUC, 6835

Qiu, Y. L. 1998b, IAUC, 6841

Schlegel, E. M. 1990, MNRAS, 244, 269

Tully, R. B. 1988, Nearby Galaxies Catalog (Cambridge University Press, Cambridge)

Turatto, M., Cappellaro, E., Danziger, I. J., et al. 1993, MNRAS, 262, 128

Wegner, G., \& Swanson, S. R. 1996, MNRAS, 278, 22 